\title{
The DØ silicon micro-strip tracker
}

\author{
M. Weber, for the D $\varnothing$ Collaboration ${ }^{a}$ \\ webermi@fnal.gov \\ ${ }^{\text {a} F e r m i l a b, ~ P . O . B o x ~ 500, ~ B a t a v i a, ~ I L-60510, ~ U S A ~}$
}

The $\mathrm{D} \varnothing$ silicon micro-strip tracker (SMT) is part of the DØ upgrade for the Tevatron RunII at Fermilab. The detector has been running successfully since the start of the RunII physics data taking. The tracking and vertexing performance match the expectation from Monte-Carlo studies. An additional inner layer (Layer0) of silicon sensors at $R=1.6 \mathrm{~cm}$ will be installed in 2005 .

\section{Introduction}

The $\mathrm{D} \varnothing$ silicon micro-strip tracker (SMT) is located inside a 2 Tesla solenoid magnet and provides track and vertex reconstruction capabilities over the pseudo-rapidity coverage $\eta$ of the $\mathrm{D} \varnothing$ detector. The impact parameter of the the reconstructed tracks is used to identify a vertex displaced from the beam interaction point (IP). This is used, for example, in Higgs search, top physics and $B$ physics. The construction was finished in December 2000, installation was completed in March 2001.

In the following we discuss the design [1], performance and operation of the DØ SMT, and the design of Layer 0.

\section{Design}

Due to the length of the luminous region at the Tevatron $(\mathrm{RMS} \simeq 25 \mathrm{~cm})$ a design with barrels intercept with discs was chosen. A drawing of the DØsilicon tracker is shown in figure 1.

The six barrels provide tracking for high $p_{\mathrm{T}}$ particles with $|\eta|<1.5$ and disk detectors for forward particles with pseudo-rapidity up to $|\eta|=3$. The barrels are $12 \mathrm{~cm}$ long and consists of 72 ladders arranged in 8 layers, with pairs of layers forming four super-layers (L1 to L4). The barrels cover the radial region from $2.7 \mathrm{~cm}$ to $9.4 \mathrm{~cm}$. L1 and L3 consist of single layer (SS) sensors for the two outer barrels (in beam direction), and double sided double metal (DSDM) technologies with $90^{\circ}$ stereo for the four central barrels. Double sided (DS) ladders with $\sim 2^{\circ}$ stereo are used

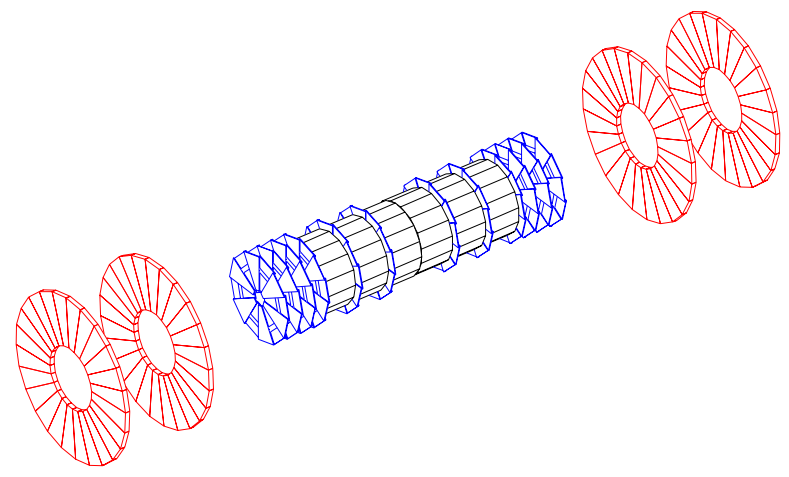

Figure 1. Isomeric view of the DØsilicon tracker. The design includes six barrels intercept with discs. There are 12 central F-discs and four forward H-discs.

for L2 and L4 in all barrels. The $12 \mathrm{~F}$-disks are located inbetween and just in front of the barrels. They are composed of 12 DS wedge $30^{\circ}$ stereo detectors. The $4 \mathrm{H}$-disks cover the most forward tracking and are composed of 24 pairs of SS detectors glued back to back. The vendors are Micron for the barrel ladders, $67 \%$ Micron and $33 \%$ Eurysis for the F-disks, and Elma for the H-disks. The number of modules is 912 in total, comprising 792,576 readout channels. Read- 
out assemblies, referred to as HDIs (High Density Interconnects), are made of Kapton flex circuits laminated to Beryllium substrates and carry the SVXIIe readout chips [2] and related components and provide bias voltage to the sensors. Each readout assembly is glued onto a silicon sensor, forming a module. The cooling system is operated at sub-atmospheric pressure and uses a mixture of $30 \%$ glycol and $70 \%$ water circulated at $-10^{\circ} \mathrm{C}$ so that the detectors run between $-5^{\circ} \mathrm{C}$ and $4^{\circ} \mathrm{C}$ when powered.

\section{Performance}

After installing and commissioning the detector, the signal to noise ratio has been measured to be $S / N=10$ to $S / N=15$ depending on the module type. The signal was taken as the cluster charge

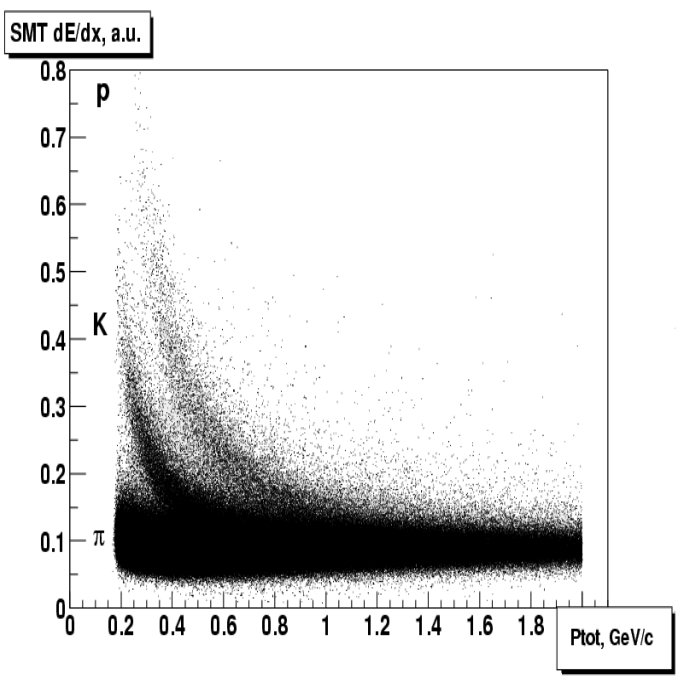

Figure 2. Energy loss as a function of momentum. One can see the separation between proton, kaon and pions. The sample used to generate the plot is enriched with kaons.

for a MIP and was corrected for the track incident angle. For the noise the RMS of the pedestal distributions was taken. The noise consists of intrin- sic (random) noise coming from the front-end of the readout chip, and environmental (coherent) noise. The signal to noise ratio calculated only from random noise is between $S / N=12$ and $S / N=18$. The signal can be used to separate protons, kaons and pions by plotting the energy loss $\mathrm{d} E / \mathrm{d} x$ as a function of the track momentum, as shown in figure 2. Figure 3 shows the track impact parameter resolution at the interaction point as a function of the transverse momentum $\left(p_{T}\right)$ of charged particles. Superimposed is the MC expectation. The $p_{T}$ dependence is caused by multi-

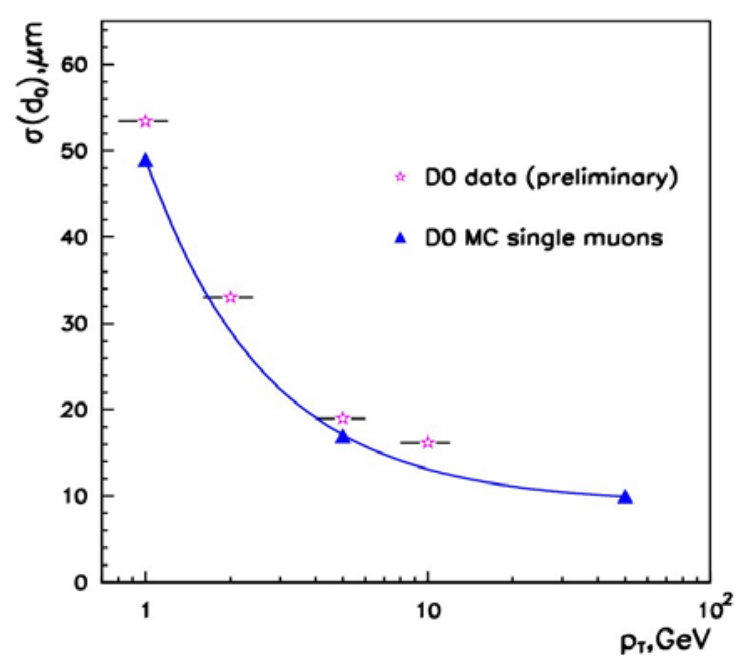

Figure 3. Impact parameter resolution $(\mu m)$ as a function of $p_{T}(\mathrm{GeV} / c)$. The stars show the results measured in data, and the triangles show the expectation from simulation.

ple scattering and hence the amount of material, while the constant term depends on the alignment. Both the $p_{T}$ dependence and constant term are in reasonable agreement between the data and MC. 


\section{Operational issues}

Currently $85 \%$ of the modules are operational. The fraction of operating modules shows a slow downwards trend, as shown in figure 4. Several

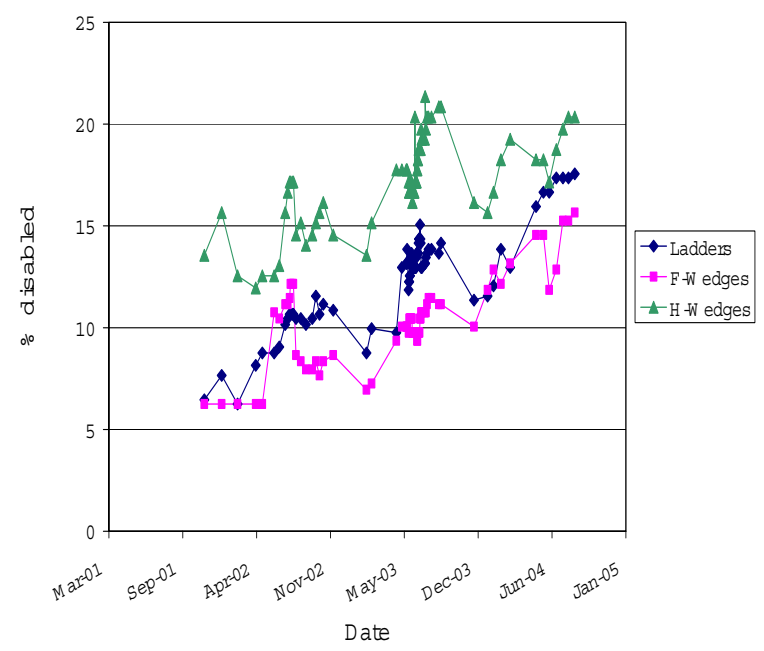

Figure 4. The fraction of disabled HDIs as a function of time. The diamonds are for ladders, the squares for F-disks, and the triangles for H-disks.

modules are usually recovered by repair work performed during shutdown periods of the accelerator. However, approximately half of the disabled modules have problems in a non-accessible area, i.e. either SVXIIe chips, HDIs, or low mass cable connecting to HDIs. Around $10 \%$ of the modules show an unstable behavior in terms of being able to include them in the readout. Some Micron F-disk sensors show breakdown-related noise starting after several months of operation. A typical example is shown in figure 5. This noise appears mostly at the p-side, and looks like microdischarges at the p-n junction, although there is no temperature dependence one would expect from micro-discharges.

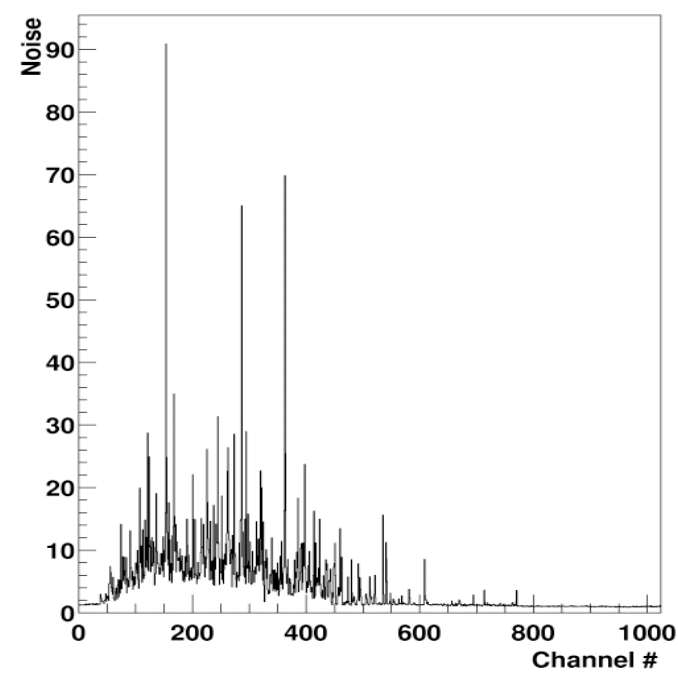

Figure 5. Noise distribution of an F-disk sensor in units of ADC counts (energy deposited by a MIP is about $25 \mathrm{ADC}$ counts).

\section{Radiation dose studies}

A reliable estimate of total absorbed dose is given by the increase in leakage current as a function of luminosity. Figure 6 shows the fluence $\Phi$ deduced from the measured leakage current increase $\Delta I$ using $\Delta I=\alpha \cdot V \cdot \Phi$, where $\alpha=2.9 \times 10^{-17} \mathrm{~A} / \mathrm{cm}$ is the damage coefficient after full annealing at $20^{\circ} \mathrm{C}$ and $V$ is the volume of the sensor. The currents have been corrected for temperature according to $I \sim T^{2} \cdot e^{(-1.23 \mathrm{eV} / k \mathrm{~T})}$. Our measured fluence of $(3.5 \pm 1.0) \times 10^{12} /\left(\mathrm{cm}^{2}\right.$ $\left.\mathrm{fb}^{-1}\right) 1 \mathrm{MeV}$ neutron equivalent at $r=2.7 \mathrm{~cm}$ is consistent with a CDF measurement from Run I of $4.1 \times 10^{12} /\left(\mathrm{cm}^{2} \mathrm{fb}^{-1}\right)$. This result is also consistent with the dose measured by a radiation monitor consisting of 16 photo-diode sensors. Radiation effects on the sensors have been studied using a $8 \mathrm{GeV}$ proton beam at the Fermilab Booster ${ }^{1}$. After each step of irradiation, the depletion voltage was measured using a $1063 \mathrm{~nm}$ laser. The depletion voltage for each detector type is shown in

$\overline{{ }^{1} \text { Data were taken at } 0,300,600,900,1500, \text { and } 2100 \mathrm{kRad}}$ 


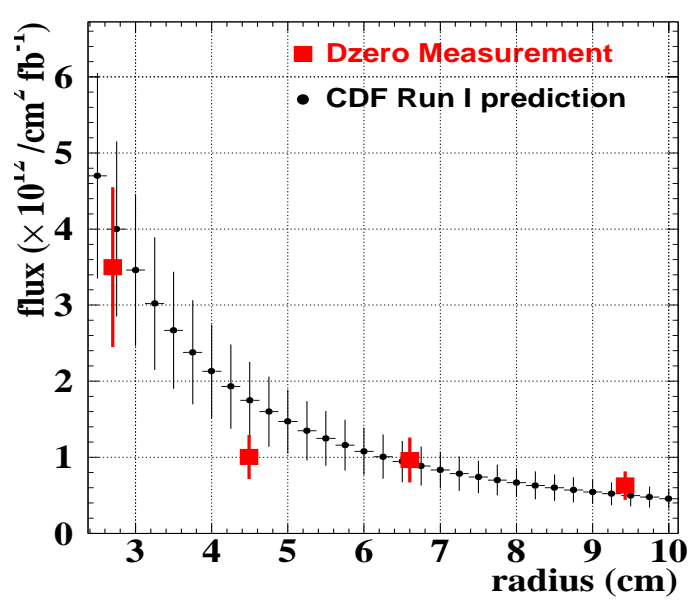

Figure 6. The measured fluence equivalent to $1 \mathrm{MeV}$ neutron as a function of distance from the IP (squares). Also shown in circles is the expectation based on CDF Run I measurement with a correction for different magnetic field.

figure 7 as a function of exposed dose. Superimposed is the expectation from the Hamburg model [4]. Also shown in figure 7 are axes for dose and luminosity ${ }^{2}$. Abnormally fast reverse annealing is observed in the DSDM detector, while the other types behaves as expected. This is considered to be due to the $1.5 \mu \mathrm{m}$ thick PECVD (plasma enhanced chemical vapor deposition) used as an insulator between the two metal layers in DSDM sensors. A test structure without such insulator does not show this abnormally fast reverse annealing. Coupling capacitor breakdown and micro-discharge are limiting the bias voltage to less than $\sim 150 \mathrm{~V}$, which will limit the lifetime of the DSDM detector in the innermost layer to an integrated luminosity of $3.5-5.0 \mathrm{fb}^{-1}$. Until now the Tevatron has delivered $0.6 \mathrm{fb}^{-1}$, but the expectation is to get $8 \mathrm{fb}^{-1}$ in RunII by 2009 .

${ }^{2}$ The respective scaling factors are $3.5 \times$ $10^{12} 1 \mathrm{MeV} \mathrm{n} /\left(\mathrm{cm}^{2} \mathrm{fb}^{-1}\right)$ and $1.8 \times 10^{13} 1 \mathrm{MeV} \mathrm{n} / \mathrm{MRad}$

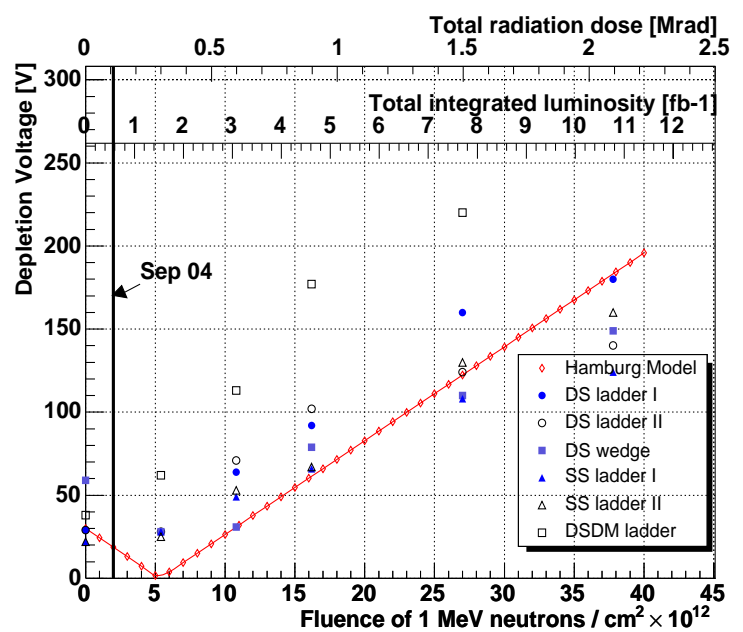

Figure 7. Depletion voltage measured as a function of fluence equivalent to $1 \mathrm{MeV}$ neutrons. The fluence is also expressed in terms of integrated luminosity in $\mathrm{fb}^{-1}$ and radiation dose in MRad at L1. The line is the expectation from Hamburg model[4]. The DSDM sensors used in L1 and L3 show abnormally fast reverse annealing.

\section{Layer0}

$\mathrm{D} \varnothing$ is building and plans to install a new layer of silicon strips (Layer0) inside the existing SMT in the fall of 2005. Layer0 consists of one layer of single sided Hamamatsu sensors directly supported by a carbon fiber structure at $1.6 \mathrm{~cm}$ from the interaction point. A six-fold geometry provides $98.5 \%$ acceptance in $\varphi$ and there are 8 sensors in $z$. Besides mitigating the tracking loss due HDI failures and to the radiation damage for the innermost layer L1, Layer0 improves the impact parameter resolution due to its proximity to the interaction point. This will improve the physics reach by increasing the $b$-jet tagging efficiency by $20 \%$. Radiation hard single sided sensor and SVX4 readout chips [3] will be used, which were originally developed for a full replacement of the current detectors ${ }^{3}$. A novel approach for the

\footnotetext{
${ }^{3}$ This upgrade has been cancelled in 2003
} 
grounding will be pursued. All the carbon fiber surface will be covered with a copper mesh. This will provide electrical contact to the carbon fibers and allow us to use the carbon fiber structure as ground. Layer0 will be read out by existing data acquisition channels taken from the outermost $\mathrm{H}$ discs, which will be removed.

\section{Conclusions}

The DØ SMT has been operational for 2.5 years and provides excellent tracking and vertexing capabilities. Operational problems include failures of readout modules, unstable modules and breakdown-related noise at F-disk sensors. Radiation studies indicate that the lifetime of the innermost layer L1 will be limited to $3.5 \mathrm{fb}^{-1}$ to $5 \mathrm{fb}^{-1}$ by micro-discharge and abnormally rapid reverse annealing of the DSDM sensor. In 2005 $\mathrm{D} \varnothing$ plans to install a new layer of silicon detectors between a new beam pipe and the innermost layer of the existing detector. This new detector will ensure good tracking and vertexing capabilities to D $\varnothing$ throughout Run II.

\section{REFERENCES}

1. E. Kajfasz, Nucl. Instrum. Method A511 16 (2003).

2. R. Yarema etal, Fermilab-TM-1892 (2001).

3. M. Garcia-Sciveres etal, Nucl. Instrum. Method A511 171 (2003).

4. G. Lindstrom etal (The RD49 Collaoration), Nucl. Instrum. Method A466 308 (2001). 